\title{
Estimated New Routing Scheme in MANETs
}

\author{
Neelesh Gupta \\ A.P., EC Dept. \\ TIEIT (TRUBA), Bhopal \\ (M.P.)-India
}

\author{
Roopam Gupta \\ HOD, IT, Dept. \\ UIT, RGPV, Bhopal \\ (M.P.)-India
}

\begin{abstract}
High probability of disappointment in Ad-Hoc networks due to energy-tired nodes is one of the main limitations. If some nodes expire early due to lack of energy, they cannot transfer the data with each other. Therefore, excessive consumption of nodes energy should be prevented. Energy conservation of a Network is a major design issue since mobile devices are often embarrassed by the battery. After suitable election of one active node among several nodes an enormous amount of energy can be saved in any local networks. This paper aims energy conservation through hexagonal GAF grid structures with the help of most popular Location-Aided Routing (LAR) scheme. This scheme uses the location of mobile node through GPS technique to improve the performance matrices such as energy saving, routing overhead and network life time in Ad-Hoc Networks.
\end{abstract}

\section{Keywords}

MANETs, Energy Conservation, Hexagonal Grids, Positionbased Routing.

\section{INTRODUCTION}

A MANET has several silent characteristics such as dynamic topologies, bandwidth constrained, limited physical security, power-constrained operation etc. These are major challenges for developing the MANET. As batteries provide limited power to the mobile nodes, thus nowadays energy is one of the most significant criteria for Ad-Hoc networks. Device can be in one of following modes such as transmission, reception, idle, or sleep states. In transmission state highest energy is consumed by the device while small energy consumption in sleep and idle state. In the idle, the radio can toggle to transmit or receive mode, which is the default state for an adhoc environment. The sleep mode has extremely low power consumption. Therefore, taking advantage of the sleep mode is very important in energy efficient protocols. In fact, nodes energy consumption should be balanced in order to increase the network life time [1]. On turning the radio off as much as possible basic principal is employed for energy conserving schemes. The performance of an Ad-Hoc network is based on employed routing scheme. Unlike cellular wireless networks, several mobile hosts need to be present in order to organize themselves into an Ad -Hoc wireless network. This conserved energy can be achieved by the proper selection of mobile node as a coordinator in wireless Ad-Hoc networks and utilize this in several applications such as route construction is called energy aware routing along with improvement in routing overload of Ad-Hoc Networks. This is aim of this paper. A virtual backbone scheme consists of special nodes are used for the energy saving algorithm and routing also presented a new distributed algorithm for constructing a connected dominating set (CDS) that is used to construct and maintain the virtual backbone of the network. Geographic Adaptive Fidelity (GAF) [2] has been proposed as an efficient power saving algorithm for wireless networks. However the properties of the grid topology in GAF have not been fully satisfied. It was shown existence of an unreachable corner in the GAF grid architecture. After investigating a couple of lossless topologies, Honeycomb virtual mesh topology (GAF-h) proposed to replace the GAF square grid. GAF-h was proved to be able to achieve zero loss with little extra cost compared to the original GAF scheme. It integrates nicely with the original GAF protocol with little computing overhead [3].

Several routing protocols, are friendly with the characteristics of Ad-Hoc networks have been proposed. In general, they can be divided into Topology-based and Position-based protocols. Topology-based routing protocols use information about links that exist in the network to perform packet forwarding. They are again divided into two categories: Proactive, Reactive protocols. Proactive routing protocols periodically broadcast control messages in an attempt to have each node always know a current route to all destinations. Proactive routing protocols are less suitable for Ad-Hoc wireless networks because they constantly consume more power throughout the network. On the other hand, reactive routing protocols are deemed more appropriate for wireless environments because they initiate a route discovery process only when data packets need to be routed. In recent developments, Position-based routing protocols exhibit better scalability, performance and robustness against frequent topological changes. Positionbased routing protocols use the geographical position of nodes through GPS to make routing in networks, which results in improving efficiency and performance [4]. The examples of these are Most Forward within Distance R (MFR), Distance Routing Effect Algorithm for Mobility (DREAM), Greedy Forwarding (GRID), and Location-Aided Routing Protocol (LAR) etc.

In these several position-based routing protocols, most popular widely used protocol is LAR. By using location information, LAR limit the scope of flood search needed to obtain a route to a destination via request zone. Locationbased routing algorithms overcome disadvantage of topologybased routing by using additional information. They require the information about the physical location of the participating nodes. Restricting the research zone reduces the route discovery packets. This protocol was designed to decrease traffic overhead generated by route discovery packets. Much research efforts have been devoted to develop energy aware routing protocols for the purpose of energy saving. This paper presents a new energy conservation scheme for multi-hop AdHoc networks. The rest of this paper is ordered as follows. Preliminaries are discussed in Section 2. Section 3 tells about GAF and its effects. Section 4 gives estimated routing scheme. Last Section concludes this paper. 


\section{PRELIMINARIES}

Several researchers had developed a number of proposals previously for energy efficient routing protocols such as BECA/AFECA, which was described by $\mathrm{Xu}$ et al. [5] powersaving approaches; Such as the Basic Energy Conserving Algorithm (BECA) and an extended version called the Adaptive Fidelity Energy Conserving Algorithm (AFECA). These approaches entail dynamically switching the nodes between sleeping, listening and active states. A technique of Span, described by Chen et al. [6] which were common for all routing protocols and they assumed that all nodes in the network are battery powered. This is not necessarily the case in real life scenarios like the ones described in the preceding examples. In the scenario using regular consumer devices the devices will be of varying strength, ranging from mobile phones to laptop computers. Ko Y. and Vaidya N. [7] described how location information may be used to reduce the routing overhead in ad hoc networks. They presented two location-aided routing (LAR) protocols. Their simulation results show that using location information routing overhead can be reduced significantly as compared to those algorithm (that does not use location information). They also suggested several optimizations on the basic LAR schemes which may improve performance.

GAF by $\mathrm{Xu} \mathrm{Y}$. [2] et al. introduced a novel power saving technique which uses location information of each node in a wireless sensor networks for grouping of nodes. This achieves power saving by making some nodes sleep autonomously. GAF groups nodes according to their locations. One active node that works as a cluster head of the group for routing is elected in each group according the remaining battery power of each node in a distributed manner. An unreachable corner in the GAF grid architecture was identified by Liu R. et al. [3] and was proved by results in an unreachable probability of 0.023 . To eliminate this unreachable probability, the scheme of reduced grid cell size, they proposed GAF-h the Honeycomb (Hexagonal) virtual mesh architecture. GAF-h is proved to be not only lossless but also comparable to the original GAF in energy conservation. They proposed an efficient honeycomb cell placement and node association algorithm. It integrates nicely with the original GAF protocol with little computing overhead. Patel A.et al. [8] identifies the unreachable corner of GAF and its effect. Unreachable corner of the cell causes the packet drop and longer path which is measured in terms of Packet Delivery Ratio and Throughput. They introduced the Hexagonal GAF algorithm and tests that with different speed and traffic. The two algorithms GAF-C and GAF-E improve the packet delivery ratio which means the less packet drop and throughput. Liu H.et al. [9] presented selective backbone construction (SBC) which starts by electing a small numbers of seed nodes in the backbone and complete its construction by making a sweep of the network spreading outwards from the seed nodes.

\section{GAF ALGORITHM}

GAF states that it locates nodes in the network and makes the best use of them to have a better fidelity. All the nodes use a location identification technique to locate itself within the network along with its nearest neighbours by using location information systems like GPS. In GAF the entire network area is divided into virtual square grids. All the nodes in the network divide themselves in virtual square grids and all those nodes which are under a same grid known as equivalent nodes with respect to forwarding packets. Nodes under a same grid coordinate among themselves to decide the sleep time interval and sequence of sleep. GAF algorithm can communicate directly to its adjacent horizontal and vertical grid cells. But the diagonal cell cannot be covered directly by virtual grid method due to range limitations.

\subsection{Unreachability in GAF}

According to the definition of a virtual grid of size $r_{G}$, the distance between two nodes at the extreme end of diagonal of any two adjacent grid cells must not be larger than $\mathrm{R}$ (radio range).Therefore the distance between nodes is,

$$
\mathrm{D}=\sqrt{\mathrm{r}_{\mathrm{G}}}{ }^{2}+\left(2 \mathrm{r}_{\mathrm{G}}\right)^{2} \quad \text { or } \mathrm{rG}_{\leq} \mathrm{R} / \sqrt{5}
$$

In GAF protocol only one node per grid is active and remaining nodes within the grid can go to sleep and save energy. Because of this the network life time increases, that depends on number of nodes per grid. For large number of nodes per grid, more nodes can go to sleep mode and further the network life time increase. To evaluate the upper bound of the lifetime achieved by GAF, $r_{\mathrm{G}}$ is set to at its maximum value $\mathrm{r}_{\mathrm{Gmax}}$

$$
\mathrm{r}_{\mathrm{Gmax}}=\mathrm{R} / \sqrt{ } 5
$$

If $\mathrm{n}$ nodes are uniformly distributed in network area $\mathrm{A}$, the minimum total number of grids given by,

$$
\mathrm{m}=5 \mathrm{R}^{2} / \mathrm{A}
$$

Thus, finally the maximum number of nodes per grid will be,

$$
\mathrm{N}_{\mathrm{G}}=\mathrm{nR}^{2} / 5 \mathrm{~A}
$$

However, some of the nodes in diagonal cell are Unreachable from some nodes in cell. Effect of this unreachable event is the data packet drop and longer path in the network. Our aim is to find out such type of algorithm which can overcome the unreachable corner problem of GAF without reducing its life time [3], [8].

\subsection{Hexagonal GAF}

Hexagonal tessellations have been used in the literature for various applications. Examples are cellular phone station placement, the representation of benzenoid hydrocarbons, computer graphics, image processing and parallel computing. The hexagon GAF grid architecture uses the hexagonal grid structure [8]. In this the square grid in GAF is replaced with a hexagon mesh as shown in Figure (1). Cell O now has six neighbours covering destinations from all directions. A Hexagon cell in GAF-h is defined as, for two adjacent cell $\mathrm{O}$ and $\mathrm{B}$, all nodes in cell $\mathrm{O}$ can communicate with all nodes in cell $\mathrm{B}$ and vice versa. The hexagon mesh has the nice property that for a cell $\mathrm{O}$, all of its six adjacent cells are at next hop. They have the same maximum distance to cell $O$. In the square grid architecture there are eight neighboring cells (four diagonal, two vertical and two horizontal cells) but only four (vertical and horizontal two each) are at next hop distance while the hexagon cell covers all six possible next hop cells with a single maximum distance due to its symmetry property. Therefore all of the next hop cells for cell $\mathrm{O}$ are equally reachable by definition. In Figure (1), if $r$ is the edge length of the hexagon then the longest distance between two adjacent cells, e.g. cells $\mathrm{O}$ and $\mathrm{B}$, is represented by line $(\mathrm{o}, \mathrm{b})$, 


$$
D_{(o, b)}=\sqrt{ } 13 r \leq R
$$

Maximum value of $r$ is given by,

$$
\mathrm{rHmax}=\mathrm{R} / \sqrt{ } 13
$$

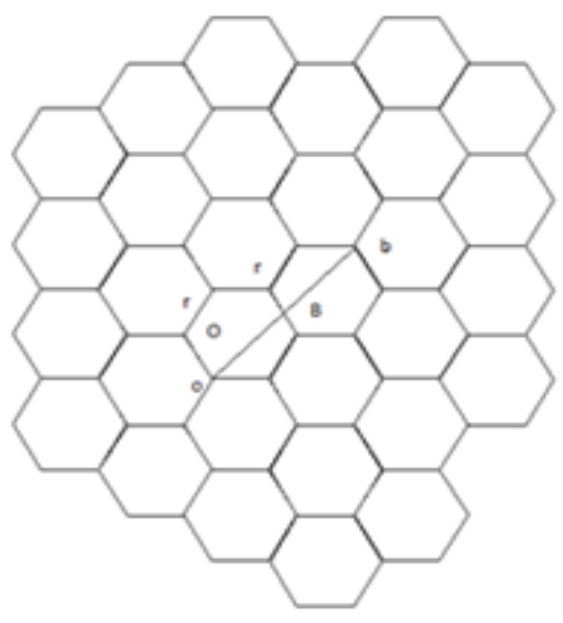

Fig.1- Hexagon GAF Grid Structure

Area of one hexagon cell is,

$$
\mathrm{S}=3 \sqrt{3} \mathrm{R} 2 / 26
$$

Finally, network size A with n nodes, the number of nodes per hexagon cell will be,

$$
\mathrm{NH}=3 \sqrt{3} \mathrm{n} \mathrm{R} 2 / 26 \mathrm{~A}
$$

Comparing this GAF-h with simple GAF from equation (4) \& (8):

$$
\mathrm{NG}-\mathrm{NH} / \mathrm{NG}=0.074 \%
$$

Here, the network lifetime with hexagonal tessellations is reduced by a negligible amount of $0.074 \%$. Now with the hexagonal structure, all next hop cells become reachable all of the time. This has removed the unreachable corner suffered by the original GAF. This improvement can be interpreted in terms of either reduced packet loss or shorter path length.

\section{ESTIMATED ROUTING}

Consecutively to save energy, it is necessary that specific number of nodes be active and other nodes are in sleep state and save the energy in network. So network lifetime will be high. The following proposed steps should be chosen for this estimated energy conservation scheme in Ad-Hoc Networks.

\subsection{Selection of Coordinator (Header Node)}

In the particular geographical region, there are several local regions containing the various mobile nodes and at a time only one node will be active in wireless Ad-Hoc Network is called, coordinator, others conserve the energy at same time. Whole liability of data passing will go towards the coordinator and complete the job without affecting the network connectivity.

\subsection{Eligibility Rule}

In this network, coordinators work as a network backbone that forwards the messages. A node should become a coordinator, if two neighbours of that node cannot arrive at each other either straightforwardly or via one or two coordinators, and each coordinator occasionally checks if it should withdraw as a coordinator.

To avoid several nodes becoming coordinators simultaneously and redundantly, a node delays announcing itself as a coordinator by a random amount of time and for particular $\mathrm{j}$ node,

It is given by:

$$
\text { Delay }=\left(\left(1-E_{r} / E_{o}\right)+\left(1-C j / N_{2}\right)+R\right) N j T
$$

Where, $\mathrm{N}_{\mathrm{j}}=$ No. of neighbors for particular node $(\mathrm{j})$,

$\mathrm{C}_{\mathrm{j}}=$ No. of additional pairs, $\mathrm{Nj}_{2}=$ No. of neighbor pair,

$\mathrm{Er}=$ Residual energy at a node

$\mathrm{E}_{\mathrm{o}}=$ Peak energy available at the same node,

$\mathrm{T}=$ Round trip delay.

However there should be fulfilling the requirement of coordinator in network for each node and also eligibility rule for the same.

\subsection{Design Steps}

The following steps must be taken:

(a) One coordinator must be selected from a specific radio range of nodes. Coordinators rotate continuously in network.

(b) A node is more likely become a coordinator with higher residual energy.

(c) There should be eligibility criteria for a coordinator and it will be moved in the network also.

(d) Coordinator will make announcement through HELLO message when it is about to change the responsibility.

Finally the selected coordinators work in network during the routing, so that energy conservation can be achieved.

\subsection{Position based Routing}

After the coordinator selection in the localized network then energy aware location-based routing is implemented, in which data go from source to destination via coordinators. LAR protocol can be categorized into position-based or location based routing protocol that is same as the flooding algorithms, with some modifications. The modification at the source is to define a request zone so that only the nodes in the request zone can be allowed to forward a request message into their neighbours. While in other on-demand protocols flooding process is done for whole Ad-Hoc Network.

\subsection{LAR}

Ko and Vaidhya (1998) proposed an optimization to route discovery known as Location-Aided Routing (LAR), that uses knowledge of the physical information of the destination node in route discovery (via GPS) to narrow the area of network over which the RREQ must be propagated. It is ondemand source routing protocol. Two Location-Aided Routing algorithms that use location information have been proposed previously .LAR assumes two regions, Expected region and Request region .LAR uses request region as a 
rectangle and expected region as a circle in shape. In this, Expected region is determined according to the location of destination and Request region is determined according to the location of source node. The LAR scheme determines a request zone. This request contains the source node $S$ and the expected region. Under LAR two approaches appear LAR-1 and LAR-2. In LAR-1, the source node $S$ depends on the expected region to determine the four corners of the request region. Node $S$ includes their coordinates with the route request message transmitted when the route discovery is initiated. When a node receives a route request, it discards the request if the node is not within the request region. For instance, if node $I$ receives the route request from another node, node $I$ forwards the request to its neighbours because it is located in the request zone. However, when node $J$ receives the route request, node $J$ discards the request, as node $J$ is not within the request zone [4], [7].

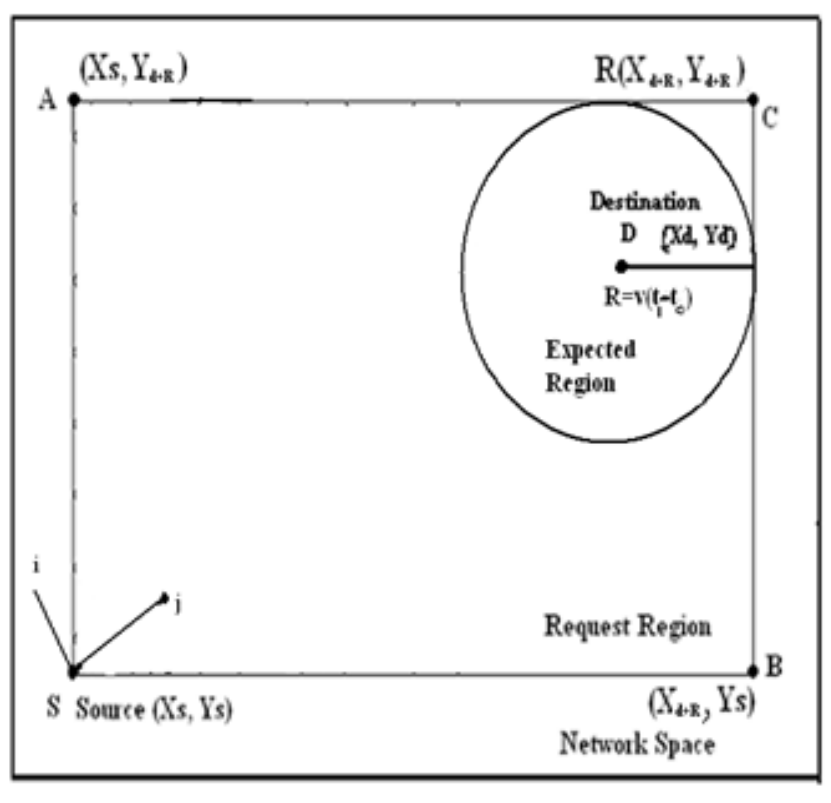

Fig. 2 - (LAR-1)

In this scheme, source node may be within or outside the expected region, if a node $\mathrm{S}$ wishes to send data to a node $\mathrm{D}$, for which it knows the prior location $\mathrm{L}$ at time $\mathrm{t}_{0}$ and speed of node $\mathrm{D}$ speed is $\mathrm{u}$, and then $\mathrm{S}$ expects that $\mathrm{D}$ will be located within an "expected zone" at time $t_{1}$, a circular area of radius $\mathrm{u}\left(\mathrm{t}_{1}-\mathrm{t}_{0}\right)$ and Centre L. If node $\mathrm{S}$ does not know the previous location L, then the "expected zone" for node D will be considered as the whole network geographical region and this algorithm will follow the basic flooding as in the DSR algorithm [8].This scheme give terminals the capability of determining whether they belong to a requested zone or not, so as to know if they should forward certain route request messages [4], [3].In this way, LAR protocols by using location information reduce the research zone where one can find a path to the destination node. This Restriction of the research zone reduces the route discovery packets in networks.

Reactive routing protocols such as, AODV, DSR, TORA, LAR execute route novelty and maintenance mechanism. LAR extend this on-demand by using physical location (GPS) of mobile nodes in the network and a significant reduction in routing overhead can be achieved even in large-scale wireless networks [10]. Better data packet delivery ratio (PDF) can be achieved for LAR at high mobile nodes speed. Thus, LAR can be effectively used when an infinite queue of data packets is to be sent in the network.

After integrating these two steps we can develop a new energy conserving technique in Ad-Hoc Networks that is shown below in fig. 3

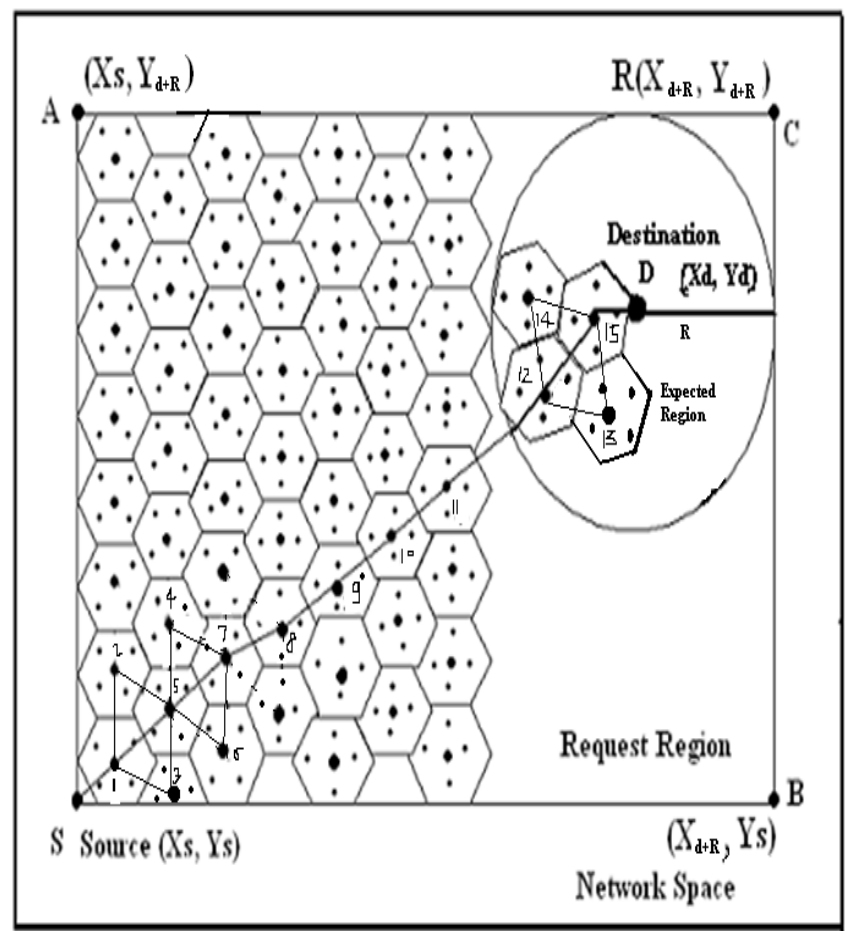

Fig. 3- Estimated Scheme

Our aim is to establish route from source to destination in particular geographical region. The radius of circular expected region will be of radius $R$ is equals to the $u\left(t_{1}-t_{0}\right)$ within request region. In this estimated technique, under LAR-1 via hexagonal tessellations routing can be established from source to destination in Ad-Hoc Networks. Serial no 1 to 15 are the main active nodes in their individual hexagonal grid and accomplished the work of routing in the networks. In each hexagonal grid, only one numbering node is active while all other nodes are in sleep mode and save their energy. In this within the request region route is established. Routing is possible within the request region through only these selected coordinators. Next broadcasting node will be selected according to the distance from the sending node. Finally in this location based routing specific key performance matrices can also be improved.

\section{CONCLUSION}

Energy conservation is one of the main tribulations in MANETs. In order to facilitate communication within an AdHoc Network, an efficient routing is required to discover routes among mobile nodes. An effective efficient energy conservation position based scheme in Networks can be invented. Using this method energy conservation can be achieved on limited level. While in the same environment compare than others, position-based routing protocol (LAR) 
can provide better results in the terms of some crucial parameters such as energy saving, routing overhead and network life time of MANETs.

Numerous optimizations on location-based scheme can be recommended, by which performance will be improve and also extend other ways of using location information in AdHoc Networks in future.

\section{REFERENCES}

[1] Akbari F., Majid S., Mahnaz N. Bonab I.,” Equalization of Energy Consumption in Ad Hoc Networks using Learning Automata", IJCSNS International Journal of Computer Science and Network Security, VOL.9 No.12, December 2009.

[2] Y. Xu, J. Heidemann, and D. Estrin, "Geography informed Energy Conservation for Ad-Hoc Routing", Proceedings of 7th Annual International Conference on Mobile Computing and Networking, pp 70-84, 2001.

[3] Ren Ping Liu, Glynn Rogers, and Sihui Zhou, "Honeycomb Architecture for Energy Conservation in Wireless Sensor Networks" IEEE Globecom, pp 1-5, 2006.

[4] Khamis L., Kiah L., Qabajeh M., “A Qualitative Comparison of Position-Based Routing Protocols for AdHoc Networks" IJCSNS International Journal of Computer Science and Network Security, VOL.9 No.2, February 2009.

[5] Kristensen M.,Bouvin N., "Energy Efficient MANET Routing Using a Combination of Span and BECA/AFECA" Journal Of Networks, Vol. 3, No. 3, March 2008.

[6] Chen B., Jamieson K., Balkrishnan H. and Morris R.,'Span: An Energy-Efficient Coordination Algorithm for Topology Maintenance in Ad Hoc Wireless Networks", ACM Wireless Networks Journal, pp-481494, Vol.8, Sept.2002.

[7] Ko Young and. Vaidya N.,"Location-Aided Routing (LAR) in Mobile Ad-Hoc Networks", Wireless Networks, Vol.6, Issue 4,307-321, July 2000.
[8] Patel A.,. Joshi R. "Energy Conservation for Wireless Mobile Ad hoc Networks using Hexagonal GAF Protocol"'2009.

[9] Liu H. , Gupta R. "Selective Backbone Construction for Topology Control in Ad Hoc Networks" IEEE International Conference on Mobile Ad-hoc and Sensor Systems, 2004.

[10] Broustis I., Jakllari G., Repantis T., a Mart Molle,"A Comprehensive Comparison of Routing Protocols for Large-Scale Wireless MANETs"IEEE 2006.

\section{AUTHORS PROFILE}

Neelesh Gupta is Pursuing Ph.D in Electronics and Communication from Rajiv Gandhi Technical University (RGTU), Bhopal (M.P.) - India. He has a rich experience of teaching in various Technical institutions of reputed in MP, India. $\mathrm{He}$ is having more than 08 years of teaching Experience. Presently he is an Assistant Professor in Truba Institute of Engineering and Information Technology (T.I.E.I.T.), Bhopal (M.P.)-India. He has earned his BE in Electronics and Telecommunication in 2002 from LNCT, Bhopal and MTech degree in Microwave and Millimeter Wave in 2007 from MANIT, Bhopal. His area of research Interests are Wireless Communication, Microwave and Digital Signal Processing. He has presented a number of research papers in various National and International conferences in India and Abroad. He has Published number of Papers in International Journals. He is Life time member of IETE, New Delhi (M.P.).

Dr. Roopam Gupta is currently working as Reader \& HOD in IT department, University Institute of Technology, RGPV, Bhopal (M.P.) - India. She received her Ph.D from Barkatullah University, Bhopal, India and M.Tech from MANIT, Bhopal (M.P.) India. She has seventeen years of teaching experience. Her research interests are in the areas of Communication and Computer Networking. She is the recipient of MPCST, Young Scientist Award; 1999.She is Life Member of IETE and ISTE. 